\title{
Potentiation of cancer immunity-inducing effect by pH-sensitive polysaccharide-modified liposomes with combination of TGF- $\beta$ type I receptor inhibitor-embedded liposomes
}

Authors:

Eiji Yuba*

Shinya Uesugi

Yuta Yoshizaki

Atsushi Harada

Kenji Kono

\section{Affiliations:}

Department of Applied Chemistry, Graduate School of Engineering, Osaka Prefecture University, 1-1 Gakuen-cho, Naka-ku, Sakai, Osaka 599-8531, Japan

\section{*Corresponding author:}

\section{Eiji Yuba}

Department of Applied Chemistry, Graduate School of Engineering,

Osaka Prefecture University, 1-1 Gakuen-cho, Naka-ku, Sakai, Osaka 599-8531, Japan

Tel.: +81-722-54-9913;

Fax: +81-722-54-9913;

E-mail: yuba@chem.osakafu-u.ac.jp

Keywords: $\mathrm{pH}$-sensitive liposome; regulatory $\mathrm{T}$ cell; TGF- $\beta$; dextran; cancer immunotherapy; dendritic cell

\section{Abstract}

Recent success of immune checkpoint inhibitors has revealed that canceling of immunosuppression in tumor microenvironments is crucially important to achieve effective cancer immunotherapy using tumor-specific cytotoxic T lymphocytes (CTLs). Transforming growth factor (TGF)- $\beta$ signaling also contributes to immunosuppression in tumors via inactivation of CTL and activation of regulatory $\mathrm{T}$ cells. The combination of the CTL induction system and blocking system of TGF- $\beta$ signaling is attempted in this study using antigen-loaded $\mathrm{pH}$-sensitive polysaccharide-modified liposome and liposome embedded SB505124: an inhibitor of TGF- $\beta$ type I receptor. 3-Methylglutarylated dextran (MGlu-Dex)-modified liposomes delivered the model antigenic protein, ovalbumin (OVA), into cytosol of dendritic cell line via $\mathrm{pH}$-responsive membrane disruption. Subcutaneous administration of these liposomes induced the regression of OVA-expressing tumor in mice. Additional administration of SB505124-embedded liposomes improved antitumor effects and survival in mice. Results show that intravenous administration of SB505124-embedded liposomes promoted the infiltration of CTL to tumor tissues significantly compared with single administration of MGlu-Dex-modified liposomes, leading to strong immunotherapeutic effects. Results of the present study demonstrate that the combination of $\mathrm{pH}$-sensitive polysaccharide-modified liposomes and SB-embedded liposomes is promising as an immunity-inducing system for cancer immunotherapy. 
Potentiation of cancer immunity-inducing effect by $\mathrm{pH}$-sensitive polysaccharide-modified liposomes with combination of TGF- $\beta$ type I receptor inhibitor-embedded liposomes

\section{Introduction}

The success of immune checkpoint inhibitors such as ipilimumab and nivolumab has garnered much attention for their use in cancer immunotherapy. Immune checkpoint inhibitors induce cancer therapeutic effects by canceling immunosuppression in tumor microenvironments. ${ }^{1,2}$ However, these antibody medicines for immune checkpoint molecules have been reported as effective only for some patients. Tumor-specific cytotoxic T lymphocytes (CTLs) are induced beforehand in these patients. ${ }^{3}$ Furthermore, high prices of these antibody medicines have presented social issues. Therefore, low-cost immunity-inducing systems that can induce tumor-specific CTLs and cancel immunosuppression in tumors are required.

Transforming growth factor- $\beta$ (TGF- $\beta$ ) is a cytokine related to immunosuppressive mechanisms by tumors. ${ }^{4,5}$ TGF- $\beta$ secreted in tumor microenvironments suppresses the activation of CTLs and natural killer (NK) cells, but it also activates regulatory $\mathrm{T}$ cells (Treg), leading to tumor exacerbation. ${ }^{4-6}$ Therefore, blocking of TGF- $\beta$ signaling in tumors is expected to improve immunosuppressive tumor microenvironments and to increase cancer therapeutic effects. Generally, antibodies that inhibit the binding of TGF- $\beta$ to its receptors or small-molecule drugs that inhibit the phosphorylation of intracellular domain of TGF- $\beta$ receptors have been used for blocking TGF- $\beta$ signaling. ${ }^{7}$ Small-molecule drugs are more beneficial from the viewpoint of cost than are antibodies. Because of the poor water solubility of small-molecule inhibitors of TGF- $\beta$ signaling such as LY364947 and SB505124, drug delivery carriers such as mesoporous silica and liposomes have been used to improve their bioavailability and biodistribution. ${ }^{8,9}$ Park et al., using liposomes having drug-loaded polymeric gels in their interior, reported the combined delivery of a small-molecule inhibitor for TGF- $\beta$ receptor and IL-2, which is a crucially important cytokine for the promotion of $\mathrm{T}$ lymphocyte growth. ${ }^{9}$ The combined delivery of both the TGF- $\beta$ signaling inhibitor and cytokine increased the NK cells and CTLs in tumors and improved the antitumor effects. Xu et al. reported another strategy for blocking TGF- $\beta$ signaling using siRNA of TGF- $\beta .{ }^{10}$ The combination of cancer peptide-loaded nanoparticles for induction of CTLs and siRNA-loaded nanoparticles for canceling immune suppression increased tumor infiltrating CD8-positive cells and decreased Treg in tumors. These earlier studies revealed the importance of the induction of cell-mediated immune response (cellular immunity) and blocking of TGF- $\beta$ signaling for effective cancer immunotherapy.

We have reported a CTL induction system using $\mathrm{pH}$-sensitive polymer-modified liposomes. ${ }^{11} \mathrm{pH}-\mathrm{Sensitive}$ polymers such as carboxylated poly(glycidol)s and polysaccharides become hydrophobic under weakly acidic $\mathrm{pH}$, which destabilizes the endosomal membrane after internalization to cells. ${ }^{12-15}$ These polymer-modified liposomes delivered a model antigenic protein, ovalbumin (OVA), to the cytosol of dendritic cells, which are the most potent professional antigen-presenting cells, ${ }^{16,17}$ by membrane fusion with endosomes. These liposomes also induced major histocompatibility complex (MHC) class I-mediated antigen presentation, resulting in induction of cellular immunity. Subcutaneous administration of these 
Potentiation of cancer immunity-inducing effect by $\mathrm{pH}$-sensitive polysaccharide-modified liposomes with combination of TGF- $\beta$ type I receptor inhibitor-embedded liposomes

polymer-modified liposomes to tumorbearing mice showed tumor regression. However, re-growth of tumors was observed in some mice. Therefore, potentiation of cancer therapeutic effects of polymermodified liposomes is necessary to achieve cancer immunotherapy using a liposomebased immunity-inducing system.

This study examined the combination of $\mathrm{pH}$-sensitive polymer-modified liposomes and inhibitors of TGF- $\beta$ signaling (Figure 1). For this purpose, SB505124, an inhibitor of
TGF- $\beta$ type I receptor, was used as a blockade of TGF- $\beta$ signaling. ${ }^{18}$ SB505124 was embedded into the lipid membrane of liposomes modified with poly(ethylene glycol) (PEG). 3-Methylglutarylated dextran (MGlu-Dex) was used as a biodegradable $\mathrm{pH}$-sensitive polymer for $\mathrm{pH}$-sensitization of liposomes. ${ }^{14}$ Here, the effects of combined delivery of SB505124 and antigen using each functional liposome on their cancer immunotherapeutic effects were investigated.

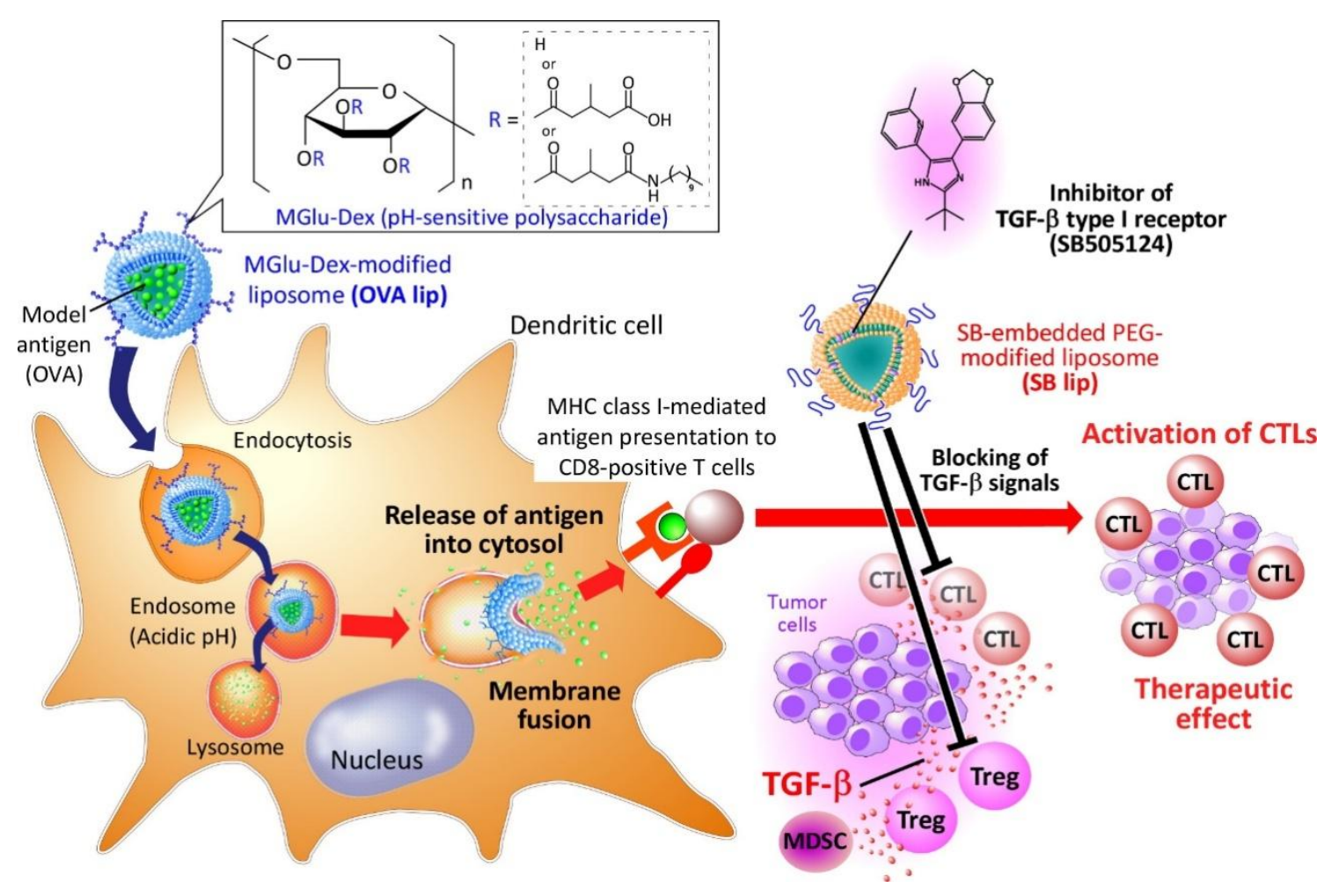

Figure 1. Concept of immunity-inducing systems using $\mathrm{pH}$-sensitive polysaccharidemodified liposomes and TGF- $\beta$ type I receptor inhibitor-embedded liposomes for CTL activation and canceling of immunosuppression. pH-Sensitive polysaccharide, MGlu-Dex-modified liposomes delivered model antigen (OVA) into cytosol of dendritic cells via $\mathrm{pH}$-responsive membrane fusion with endosomes, which induces MHC class I-mediated antigen presentation and the induction of CTLs. Furthermore, SB-embedded PEG-modified liposomes suppress the activation of regulatory $\mathrm{T}$ cells (Treg) or the inactivation of CTL via blocking the TGF- $\beta$ signaling, leading to activation of cancer immunity. 
Potentiation of cancer immunity-inducing effect by $\mathrm{pH}$-sensitive polysaccharide-modified liposomes with combination of TGF- $\beta$ type I receptor inhibitor-embedded liposomes

\section{Materials and Methods}

\subsection{Materials}

For this study, egg yolk phosphatidylcholine (EYPC), hydrogenated soy phosphatidylcholine (HSPC), and $\mathrm{N}$-(methoxypolyethyleneglycol-carbamoyl) distearoylphosphatidylethanolamine (DSPEPEG2k) were kindly donated by NOF Corp. (Tokyo, Japan). Ovalbumin (OVA), monophosphoryl lipid A (MPLA), cholesterol, 2-(5-benzo[1,3]dioxol-5-yl-2-tert-butyl-3H-i midazol-4-yl)-6-methylpyridine hydrochloride (SB505124, Figure 1), hyaluronidase type $\mathrm{V}$, deoxyribonuclease I from bovine pancreas (DNase I), and bovine serum albumin (BSA) were purchased from Sigma-Aldrich Corp. (St. Louis, MO.). Collagenase A was purchased from Roche Diagnostics Corp. (Tokyo, Japan). pH-Sensitive dextran derivatives (MGlu-Dex, Figure 1) were prepared as described in an earlier report. ${ }^{14}$ The ratios of hydroxy units, MGlu units, and decyl amide units for MGlu-Dex were 25/67/8, as estimated using ${ }^{1} \mathrm{H}$ NMR. ${ }^{14}$ E.G7-OVA, which is a chicken egg OVA gene-transfected clone of C57BL/6 mice-derived $\mathrm{T}$ lymphoma and which presents OVA with MHC class I molecules, was obtained from the American Type Culture Collection (Manassas, VA). It was grown at $37{ }^{\circ} \mathrm{C}$ in RPMI-1640 (Nacalai Tesque Inc.) supplemented with 10\% FBS (MP Biomedicals), $2 \mathrm{mM}$ L-glutamine (Wako Pure Chemical Industries Ltd.), 10 mM Hepes (Nacalai Tesque Inc.), $1 \mathrm{mM}$ sodium pyruvate (Gibco Industries Inc.), 50 $\mu \mathrm{M}$ 2-mercaptoethanol (Gibco Industries Inc.), $0.5 \mathrm{mg} / \mathrm{mL}$ G418 (Nacalai Tesque Inc.), and antibiotics. ${ }^{19}$

\subsection{Preparation of MGlu-Dex- modified liposomes (OVA lip)}

To a dry, thin membrane of EYPC (10 mg), MGlu-Dex (4.28 mg), and MPLA $(50 \mu \mathrm{g})$ was added $1.0 \mathrm{~mL}$ of OVA/PBS solution ( $\mathrm{pH} 7.4,4 \mathrm{mg} / \mathrm{mL})$. Then the mixture was vortexed at $4{ }^{\circ} \mathrm{C}$. The liposome suspension was hydrated further by freezing and thawing, and was extruded through a polycarbonate membrane with $100 \mathrm{~nm}$ pore size. The liposome suspension was centrifuged at 55,000 rpm for $2 \mathrm{~h}$ at $4{ }^{\circ} \mathrm{C}$ twice and was filtrated through $0.45 \mu \mathrm{m}$ membrane filter. Lipid and OVA concentrations were determined respectively using Test-Wako C (Wako Pure Chemical Industries Ltd.) and Coomassie (Bradford) Protein assay reagent (Thermo Scientific). The OVA concentration was adjusted to 1 $\mathrm{mg} / \mathrm{mL}$ or $0.1 \mathrm{mg} / \mathrm{mL}$ before administration.

\subsection{Preparation of SB505124- embedded liposomes (SB lip)}

To a dry, thin membrane of HSPC (11.5 mg), cholesterol (5.1 $\mathrm{mg})$, DSPE-PEG2k (3.4 mg), and SB505124 (2.2 $\mathrm{mg}$ ) was added $2.5 \mathrm{~mL}$ of PBS (pH 7.4). The mixture was dispersed by 15 min sonication at $60{ }^{\circ} \mathrm{C}$. The liposome suspension, which was hydrated further by freezing and thawing, was extruded through a polycarbonate membrane with $100 \mathrm{~nm}$ pore size at $60{ }^{\circ} \mathrm{C}$. The liposome suspension was centrifuged twice at 55,000 rpm for $2 \mathrm{~h}$ at $4{ }^{\circ} \mathrm{C}$ and was filtrated through a $0.45 \mu \mathrm{m}$ membrane filter. The lipid concentration was found using Test-Wako $\mathrm{C}$ and was adjusted to $1.1 \mathrm{mM}$ before administration. 
Potentiation of cancer immunity-inducing effect by $\mathrm{pH}$-sensitive polysaccharide-modified liposomes with combination of TGF- $\beta$ type I receptor inhibitor-embedded liposomes

\subsection{Characterization of liposomes}

Diameters and zeta potentials of the liposomes $(0.1 \mathrm{mM}$ of lipid concentration) were measured (Zetasizer Nano ZS ZEN3600; Malvern Instruments, Ltd., Worcestershire, UK). Data were obtained as averages of more than three measurements of different samples. The $\mathrm{pH}$ sensitivity of MGlu-Dex-modified liposomes was evaluated using fluorescence dye pyranine-loaded liposomes instead of OVA, as described in earlier reports of the literature (Figure S1). ${ }^{14}$ Cellular association and intracellular distribution of MGlu-Dexmodified liposomes in a murine dendritic cell-derived DC2.4 cell $^{20}$ were examined using lipophilic fluorescence dye (DiI; Life Technologies Inc.) and FITC-labeled OVA, as described in earlier reports (Figure S2). ${ }^{14}$

\subsection{Treatment of tumor-bearing mice with liposomes}

Female C57BL/6 mice (H-2 ${ }^{\mathrm{b}}, 7$ weeks old) were purchased from Oriental Yeast Co. Ltd. (Tokyo, Japan). Experiments were conducted in accordance with the Osaka Prefecture University guidelines for animal experimentation.

E.G7-OVA cells $\left(1 \times 10^{6}\right.$ cells $)$ were inoculated subcutaneously into the backs of C57BL/6 mice under anesthesia with isoflurane. On days 7 and/or 14, 10 or $100 \mu \mathrm{g}$ of OVA-loaded MGlu-Dex-modified liposomes (OVA lip, $100 \mu \mathrm{L}$ ) was injected subcutaneously into the backs of the mice under anesthesia. Additionally, SB505124-embedded liposomes (SB lip, 100 $\mu \mathrm{L}, 1.1 \mathrm{mM}$ lipids) were injected to the tumor or via tail vein at pre-determined times (as shown in each figure caption). Tumor sizes were monitored from the day of inoculation. Mice immunized subcutaneously with PBS were used as controls to confirm cancer development following the inoculation with E.G7-OVA cells. Mice were killed when tumor volumes became greater than $2,500 \mathrm{~mm}^{3}$ or when they had survived longer than 50 days. All treated groups included 4-9 mice.

\subsection{Preparation of tumor-derived single cells}

E.G7-OVA cells $\left(2 \times 10^{6}\right.$ cells $)$ were inoculated subcutaneously into the left back of each C57BL/6 mouse under anesthesia. On day 7, $10 \mu \mathrm{g}$ of OVA-containing OVA lip was injected subcutaneously into the right back of each mouse under anesthesia with isoflurane. In addition, SB lip $(100 \mu \mathrm{L}, 1.1$ $\mathrm{mM}$ lipids) was administered intratumorally or intravenously. On days 10 and 14, tumors of the respective mice were excised and were minced into small pieces. Then they were immersed in RPMI1640 containing 5\% FBS, $0.5 \mathrm{mg} / \mathrm{mL}$ collagenase A, $0.2 \mathrm{mg} / \mathrm{mL}$ hyaluronidase type $\mathrm{V}$, and $0.02 \mathrm{mg} / \mathrm{mL}$ DNase I for $2 \mathrm{~h}$ at $37^{\circ} \mathrm{C}$ with rotation (Multi Bio RS-24; Biosan, Riga, Latvia). Cell suspensions were filtered through $70 \mu \mathrm{m}$ cell strainers (Corning Inc.) and were washed twice with RPMI1640 containing 5\% FBS. Finally, the red blood cells were lysed using an ammonium chloride/tris solution. The cells were washed twice with staining buffer (PBS containing $0.1 \%$ BSA and $0.01 \%$ sodium azide).

\subsection{Detection of Foxp3-positive cells in tumor \\ For intracellular staining, tumor- derived single cells were fixed and}


Potentiation of cancer immunity-inducing effect by $\mathrm{pH}$-sensitive polysaccharide-modified liposomes with combination of TGF- $\beta$ type I receptor inhibitor-embedded liposomes

permeated using Foxp3/Transcription Factor Staining Buffer Set according the manufacturer's instructions (eBioscience). Cells $\left(2 \times 10^{6} / 100 \mu \mathrm{L}\right)$ were then incubated with $0.5 \mu \mathrm{g}$ of anti-mouse $\mathrm{CD} 16 / 32$ (eBioscience) for $15 \mathrm{~min}$ on ice to block nonspecific binding of the subsequently used antibody reagents. The cells $\left(1 \times 10^{6} / 100 \mu \mathrm{L}\right)$ were incubated with $1.0 \mu \mathrm{g}$ of anti-mouse/rat Foxp3 antibody labeled with phycoerythrin (PE) (FJK-16s; eBioscience) for $1 \mathrm{~h}$ on ice. Then, stained cells were analyzed using a flow cytometer (CytoFlex; Beckman Coulter Inc.). Between all incubation steps, cells were washed twice with permeabilization buffer of Foxp3/Transcription Factor Staining Buffer Set.

\subsection{Detection of CD8-positive cells in} tumor

Tumor-derived single cells $(2 \times$ $10^{6} / 100 \mu \mathrm{L}$ ) were incubated with $0.5 \mu \mathrm{g}$ of anti-mouse CD16/32 for $15 \mathrm{~min}$ on ice. After washing, the cells $\left(1 \times 10^{6} / 100 \mu \mathrm{L}\right)$ were incubated with $0.05 \mu \mathrm{g}$ of anti-mouse CD8 $\alpha$ antibody labeled with PE (53-6.7; Exbio) for $30 \mathrm{~min}$ on ice. Then, stained cells were analyzed using a flow cytometer. Between incubation steps, cells were washed twice with staining buffer.

\subsection{Immunofluorescence staining of tumor sections}

For immunofluorescence staining, excised tumor tissues were frozen immediately after embedding in Tissue-Tek OCT (Sakura Finetek Inc.). Tumor tissues were sectioned into $10 \mu \mathrm{m}$ slices using a cryomicrotome (CM1520; Leica) and were mounted on glass slides. The sections were fixed using acetone/methanol and were incubated with PBS containing anti-mouse CD16/32 and 10\% goat serum (The Jackson Laboratory) for $30 \mathrm{~min}$ at room temperature to block the nonspecific binding of antibody. Subsequently, the sections were incubated with anti-mouse/rat CD8 $\alpha$ antibody labeled with Alexa Fluor488 (1:100 dilution, 53-6.7, $\mathrm{BD}$ Biosciences) overnight at room temperature. Slides were mounted with Vectashield containing 4, 6-diamidino2-phenylindole (DAPI; Vector Laboratories Inc.). Confocal laser scanning microscopic (CLSM, LSM 5 Exciter; Carl Zeiss Inc.) analysis was applied to these slides.

\subsection{Statistical Analysis}

Student's $t$-test was used for statistical evaluation of the results presented in Figure S2. Survival analysis using the Log-rank test was performed as shown in Figures 2 and 4. Results are presented in Tables S1 and S2.

\section{Results and Discussion}

\subsection{Preparation of liposomes}

For this study, the combination of antigen delivery by $\mathrm{pH}$-sensitive polysaccharide-modified liposomes and canceling of immunosuppression by TGF- $\beta$ receptor inhibitor-embedded liposomes were examined (Figure 1). As an intracellular delivery carrier of model antigen (OVA), $\mathrm{pH}$-sensitive polysaccharide, 3-methylglutarylated dextran (MGlu-Dex)-modified liposome was used. Table 1 presents the size and zeta potential of EYPC liposomes modified with and without MGlu-Dex. Both liposomes exhibited size of around $100 \mathrm{~nm}$, which corresponds to the pore size of polycarbonate membrane during extrusion. The zeta potential of MGlu-Dex-modified liposomes 
Potentiation of cancer immunity-inducing effect by $\mathrm{pH}$-sensitive polysaccharide-modified liposomes with combination of TGF- $\beta$ type I receptor inhibitor-embedded liposomes

became more negative than that of unmodified EYPC liposome, indicating that MGlu-Dex having carboxy groups bound onto the liposomal membrane. The pH-responsive content release properties were investigated using fluorescent dye (pyranine)-loaded liposomes (Figure S1). Unmodified liposomes showed no content release at any $\mathrm{pH}$. MGlu-Dex-modified liposome was stable at neutral $\mathrm{pH}$, but it exhibited significant content release below $\mathrm{pH}$ 6, which corresponds to endosomal $\mathrm{pH}$ inside of cells. ${ }^{21}$ Reportedly, carboxy groups of MGlu-Dex are deprotonated at neutral $\mathrm{pH}$; the interaction of MGlu-Dex with lipid membrane is low. In contrast, MGlu-Dex became hydrophobic after protonation of carboxy groups at weakly acidic $\mathrm{pH}$, which induces membrane disruption. ${ }^{14}$ Such properties of MGlu-Dex-modified liposomes are beneficial for the intracellular delivery of contents. Actually, MGlu-Dex-modified liposomes delivered FITC-OVA into cytosol of the dendritic cell line more efficiently than unmodified liposomes did (Figures S2A and $\mathrm{S} 2 \mathrm{~B})$. This result derives from membrane disruption of endosomes by MGlu-Dex responding to acidic $\mathrm{pH}$ in endosomes. In addition, MGlu-Dex-modified liposomes were taken up by dendritic cells more efficiently than unmodified liposomes were (Figure S2C). Therefore, MGlu-Dexmodified liposomes are expected to induce OVA-specific cellular immunity by efficient cytoplasmic delivery of OVA to antigenpresenting cells.

Table 1. Particle Sizes and Zeta Potentials of Liposomes

\begin{tabular}{llc}
\hline Liposome & Size $(\mathrm{nm})$ & Zeta potential $(\mathrm{mV})$ \\
\hline Unmodified liposome & $135 \pm 3$ & $-10 \pm 5$ \\
MGlu-Dex-modified liposome & $104 \pm 1$ & $-33 \pm 1$ \\
SB-embedded PEG-modified liposome & $114 \pm 15$ & - \\
\hline
\end{tabular}

Because SB505124, an inhibitor of TGF- $\beta$ receptor, is a hydrophobic drug, the delivery of SB505124 was attempted using SB505124-embedded liposomes inside of lipid membrane. SB505124 was introduced to the liposomes composed of HSPC, cholesterol, and DSPE-PEG, which is the same lipid composition with stealth liposome or commercially available Doxil ${ }^{22}{ }^{22}$ The SB505124-embedded liposome size was 114 $\mathrm{nm}$, which is almost equal size to the pore size of polycarbonate membrane used for extrusion (Table 1).

\subsection{Cancer therapeutic effect of the} combination of OVA lip and SB lip

The antitumor effects against tumor-bearing mice were examined using OVA-loaded MGlu-Dex-modified liposomes (OVA lip) and SB505124-embedded liposomes (SB lip) (Figure 2). After inoculation of OVA-expressing tumor cells (E.G7-OVA cells) to mice, the tumor grew significantly in PBS-treated mice. Tumor volumes reached over $2,500 \mathrm{~mm}^{3}$ within 20 days (Figure 2A). In contrast, the subcutaneous administration of OVA lip strongly suppressed tumor growth and 
Potentiation of cancer immunity-inducing effect by $\mathrm{pH}$-sensitive polysaccharide-modified liposomes with combination of TGF- $\beta$ type I receptor inhibitor-embedded liposomes

decreased tumor volumes until 17 days after tumor inoculation (Figure 2C). This result is consistent with those we have reported earlier. $^{14}$ It indicates that cytoplasmic delivery of OVA to dendritic cells by OVA lip (Figure S2B) induced OVA-specific cellular immunity. However, tumors re-grew after Days 17-19 (Figure 2C), which suggests that cellular immunity might be depleted in immunosuppressive environments of tumor. TGF- $\beta$ signaling plays a crucially important role in suppressing the activation of CTLs and in activating Treg. $^{4-6}$ Therefore, the combination of inhibitor of TGF- $\beta$ signaling (SB505124) with OVA lip was examined. The intratumoral administration of SB lip following subcutaneous administration of OVA lip improved antitumor effects (Figure 2D) and the survival of mice (Figure 2E) more than single administration of OVA lip did. Single administration of SB lip to the tumor showed no antitumor effects under experimental conditions (Figure 2B). Therefore, the combination of OVA lip and SB lip might be important for antitumor immunity prolongation.
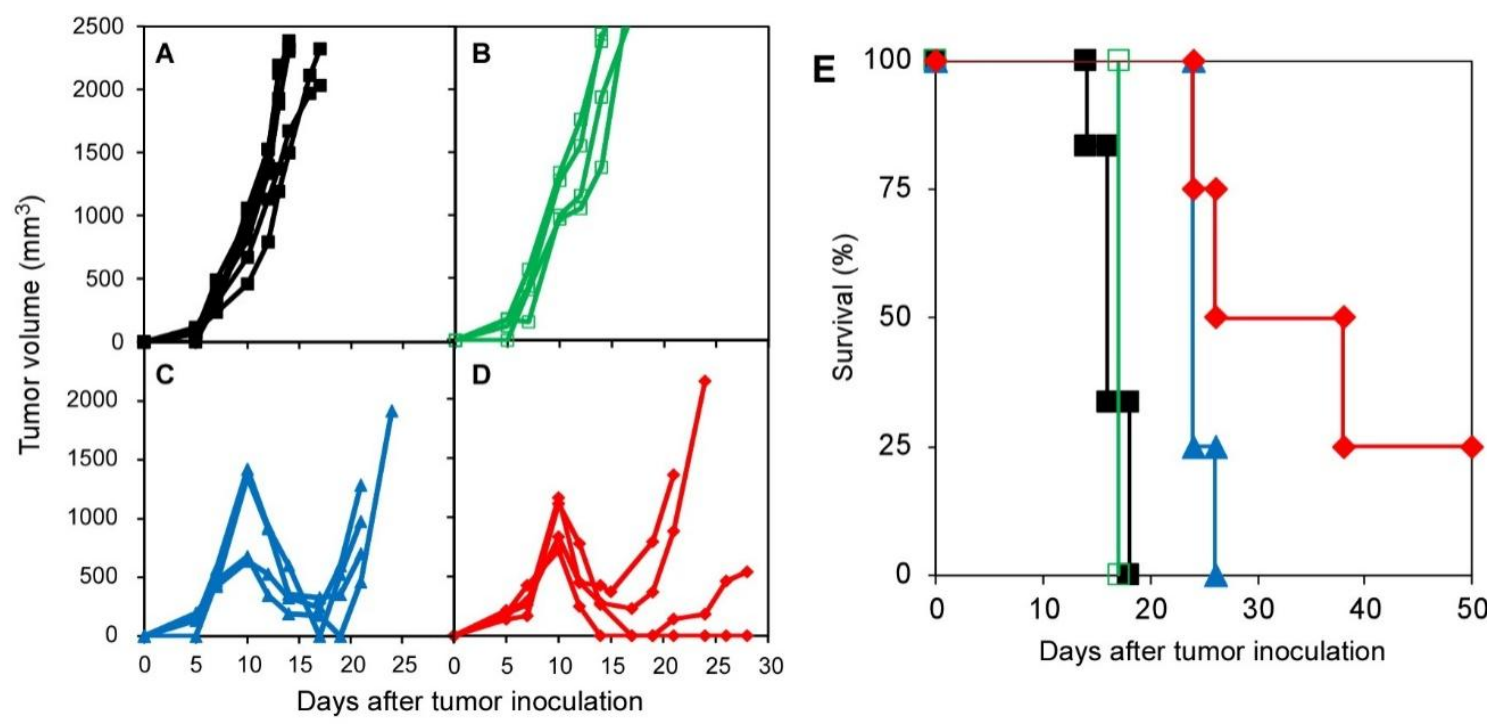

Figure 2. Antitumor effects induced by administration of OVA-loaded liposomes modified with MGlu-Dex (OVA lip) and SB505124-embedded liposomes (SB lip). E.G7-OVA cells $\left(1 \times 10^{6}\right.$ cells/mouse) were transplanted subcutaneously to the left back of C57BL/6. Then the tumor volume was followed. OVA lip containing $100 \mu \mathrm{g}$ of OVA was injected subcutaneously into the right back of each mouse. Then $100 \mu \mathrm{L}$ of SB lip (1.1 mM lipids) was administered into the tumor at days 7 and 14 after tumor inoculation. Individual tumor volumes (A-D) and survival (\%) (E) of mice treated with PBS (A, closed squares), SB lip (B, open squares), OVA lip (C, triangles), and both OVA lip and SB lip (D, diamonds) were followed from tumor cell inoculation. Mice were killed when the tumor volume exceeded 2,500 $\mathrm{mm}^{3}$. 
Potentiation of cancer immunity-inducing effect by $\mathrm{pH}$-sensitive polysaccharide-modified liposomes with combination of TGF- $\beta$ type I receptor inhibitor-embedded liposomes

Nevertheless, no significant difference between OVA lip and OVA lip/SB lip combination was found for mice survival ( $p=.0979$, Table S1), probably because individual differences of mice were considerable under these experimental conditions. Therefore, the number of mice increased. Two tumor burdens were inoculated to both sides of mice to elucidate the SB lip immunity induction mechanism (Figure 3). In these experiments, PBS and OVA lip were administered subcutaneously to different sites with left and right tumors, whereas SB lip was injected only to the left tumor, as shown in Figure 3. In the case of PBS-treated mice, tumor volumes of almost all mice reached the endpoint $\left(2,500 \mathrm{~mm}^{3}\right)$ within 20 days (Figure 3A). Similarly to that shown in Figure 2, subcutaneous administration of OVA lip suppressed tumor growth. Some mice became tumor-free, but no significant difference was found between the growths of left and right tumors (Figure 3B), which indicates that OVA-specific CTLs induced by OVA lip attacked both left and right tumors equally. In the case of OVA lip/SB lip combination, the right tumor growth was almost identical to the case of OVA lip-treated mice (Figure 3C). The left tumor growth was suppressed strongly: $78 \%$ of the left tumor and $56 \%$ of the right tumor disappeared. These results suggest the following mechanism: the administration of OVA lip induced cellular immunity. OVA-specific CTLs attacked both the left and right tumor, but CTLs in the right tumor were depleted after 20 days. In contrast, CTL activity might have been prolonged in the left tumor because of the canceling of TGF- $\beta$ signaling by SB lip administration, resulting in the improvement of antitumor effects.

\subsection{Effects of SB lip administration routes on antitumor effects}

The combination of OVA lip and SB lip actually induced synergistic antitumor effects, but intratumoral administration is restricted to superficial tumors. In addition, TGF- $\beta$ secreted from tumors spread to the body, which induces systemic immunosuppression. $^{23}$ Therefore, intravenous administration of SB lip was attempted for the combination with OVA lip because PEG-modified nano-sized liposomes circulate systemically and accumulate efficiently to the tumor site by enhanced permeation and retention effects (EPR effects). ${ }^{22,24,25}$ According to earlier reports of the literature, $38 \%$ and $13 \%$ of PEG-modified liposomes remain in the blood, respectively, at $24 \mathrm{~h}$ and $48 \mathrm{~h}$ after intravenous administration. ${ }^{26}$ Therefore, to maintain the concentration of SB505124 in the blood, SB lip was administered every 3 days (on Days 7, 10, and 13). In addition, the dose of OVA decreased to $10 \mu \mathrm{g}$. OVA lip was administered only on Day 7 in the following experiments because $56-67 \%$ of mice were cured completely by administration (twice) of $100 \mu \mathrm{g}$ OVA-containing OVA lip (Figure 3B). Distinguishing the differences between OVA lip and the OVA lip/SB lip combination is difficult. Figure 4 depicts the tumor volume change and survival of mice treated with OVA lip or SB lip, as shown in the illustration. Even at a low dose of OVA, subcutaneous administration of OVA lip induced antitumor effects and significant prolongation of survival of mice compared with PBS-treated mice (Figures 4 and S3, Table S2, $p=.00175$ ). 
Potentiation of cancer immunity-inducing effect by $\mathrm{pH}$-sensitive polysaccharide-modified liposomes with combination of TGF- $\beta$ type I receptor inhibitor-embedded liposomes

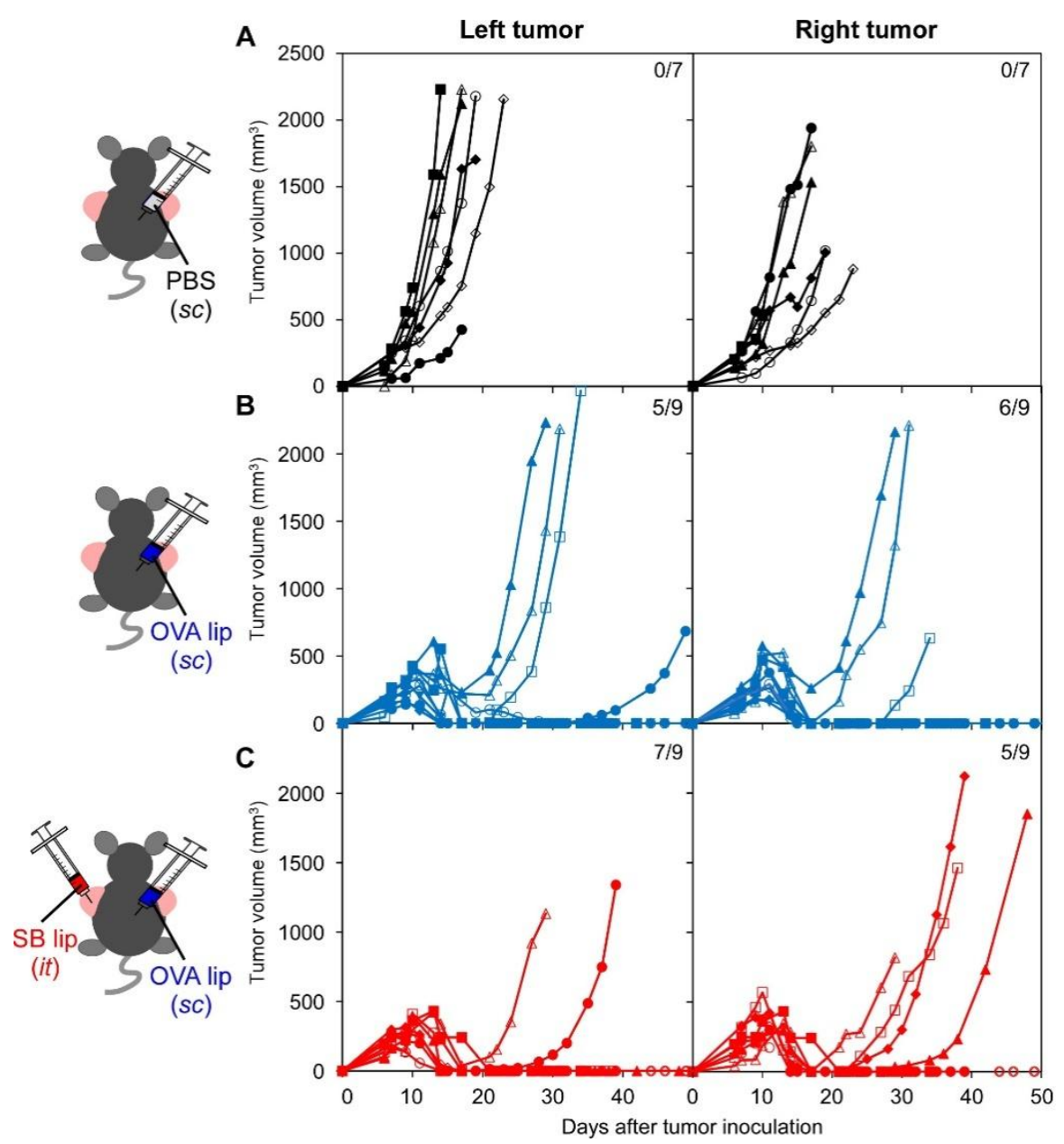

Figure 3. Individual tumor volumes of C57BL/6 mice treated with PBS (A), OVA lip (B), and both OVA lip and SB lip (C). E.G7-OVA cells $\left(1 \times 10^{6}\right.$ cells/site) were transplanted subcutaneously to the left and right backs of mice. Tumor volume was followed. OVA lip containing $100 \mu \mathrm{g}$ of OVA was injected subcutaneously into the back of each mouse, as shown in the illustration. Then $100 \mu \mathrm{L}$ of SB lip (1.1 mM lipids) was administered into the left tumor at days 7 and 14 after tumor inoculation. Mice were killed when the tumor volume exceeded 2,500 $\mathrm{mm}^{3}$. Numbers in the upper right corner represent the numbers of tumor-free mice/total mice.

The combination of intratumoral administration of SB lip and OVA lip slightly but not significantly improved survival compared with OVA lip (Figure 4B and Table S2, $p=.835$ ). In contrast, the combination of intravenous administration of SB lip with OVA lip strongly improved antitumor effects (Figure 4A) and significantly prolonged mice survival (Figure 4B and Table S2, $p=.00701)$. Single administration of SB lip showed merely antitumor effects. Therefore, the combination of intravenous administration of SB lip with OVA lip is likely to be effective to obtain the synergy of CTL induction by OVA lip and canceling of immunosuppression. Compared with intratumoral administration, SB505124embedded liposomes might be distributed not only at tumor sites but also in other organs such as the spleen or in the blood. Such a 
Potentiation of cancer immunity-inducing effect by $\mathrm{pH}$-sensitive polysaccharide-modified liposomes with combination of TGF- $\beta$ type I receptor inhibitor-embedded liposomes

difference in the biodistribution of SB505124 tumor-infiltrating lymphocytes and tumor might induce the effective activation of antitumor immunity. Therefore, to elucidate sections were analyzed using the experiments the mechanism of inducing antitumor effects, described below.
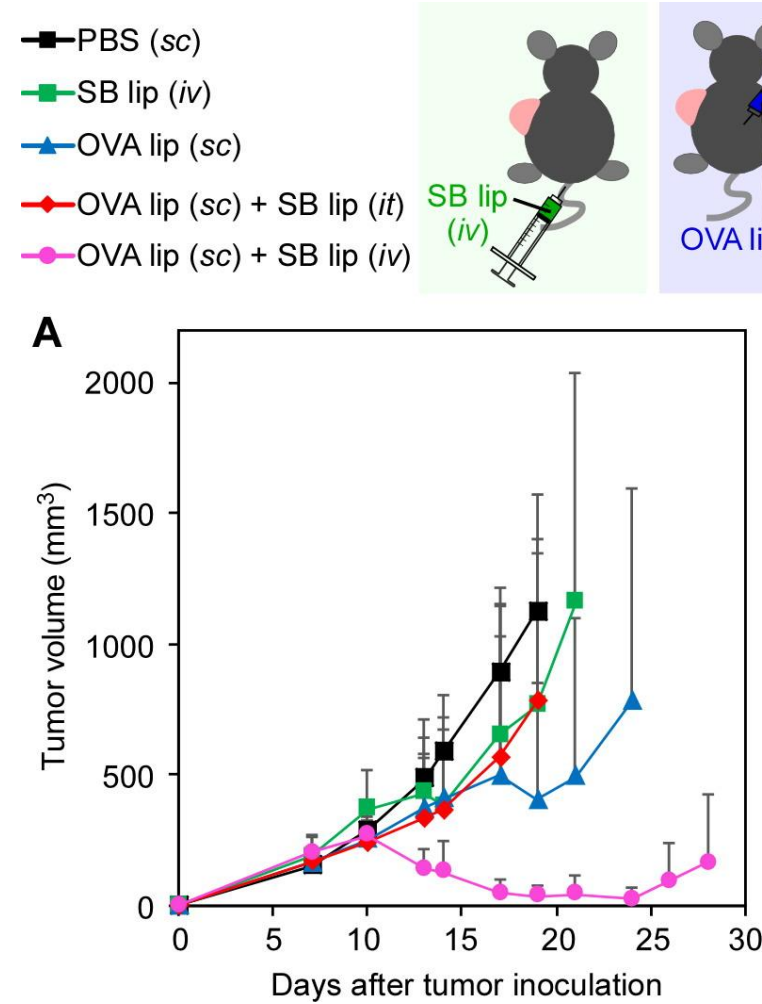
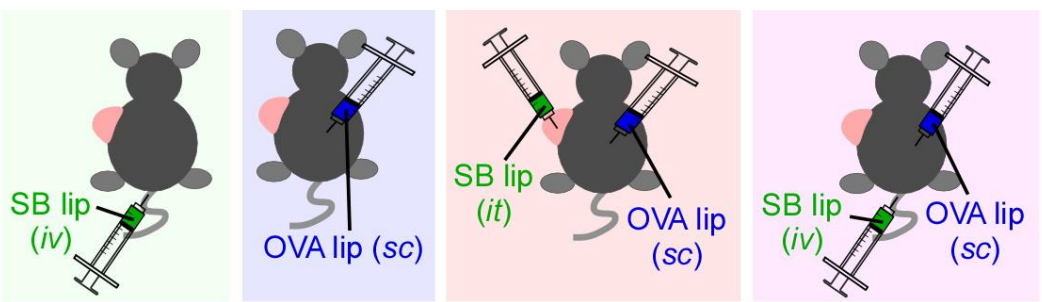

B

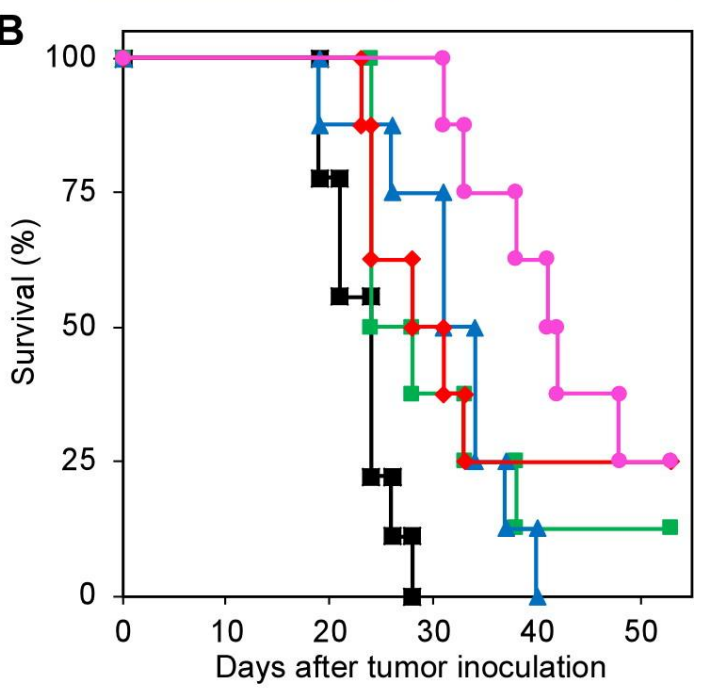

Figure 4. Antitumor effects induced by combination of OVA lip and SB lip. E.G7-OVA cells $\left(1 \times 10^{6}\right.$ cells/mouse) were transplanted subcutaneously into the left back of C57BL/6 mice. Then, the tumor volume was monitored. OVA lip containing $10 \mu \mathrm{g}$ of OVA was injected subcutaneously to the back of mice at day 7 . Then, $100 \mu \mathrm{L}$ of SB lip (1.1 mM lipids) was injected intratumorally or intravenously on days 7, 10, and 13 after tumor inoculation, as shown in the top illustration. Tumor volumes (A) and survival (\%) (B) of tumor-bearing mice were followed from tumor cell inoculation. Mice were killed when the tumor volume exceeded 2,500 $\mathrm{mm}^{3}$.

\subsection{Analysis of tumor-infiltrating} lymphocytes

SB505124 inhibits the phosphorylation of the intracellular part of TGF- $\beta$ receptor and suppresses the activation of CTL and activates Treg. ${ }^{9,18}$ Therefore, SB505124-embedded liposomes might affect the percentages of CTL or Treg in tumors. Tumors excised from mice and tumor-derived single cells were prepa- red by enzymatic digestion of extracellular matrix. Figure 5 presents the percentage of Foxp3-positive cells in tumor, which represents the Foxp3-positive activated Treg. Irrespective of the treatment group and day, the percentages of Foxp3-positive cells were almost equal. This result suggests that administration of OVA lip and/or SB lip only slightly affected the infiltration of Treg in tumor tissue. 
Potentiation of cancer immunity-inducing effect by $\mathrm{pH}$-sensitive polysaccharide-modified liposomes with combination of TGF- $\beta$ type I receptor inhibitor-embedded liposomes

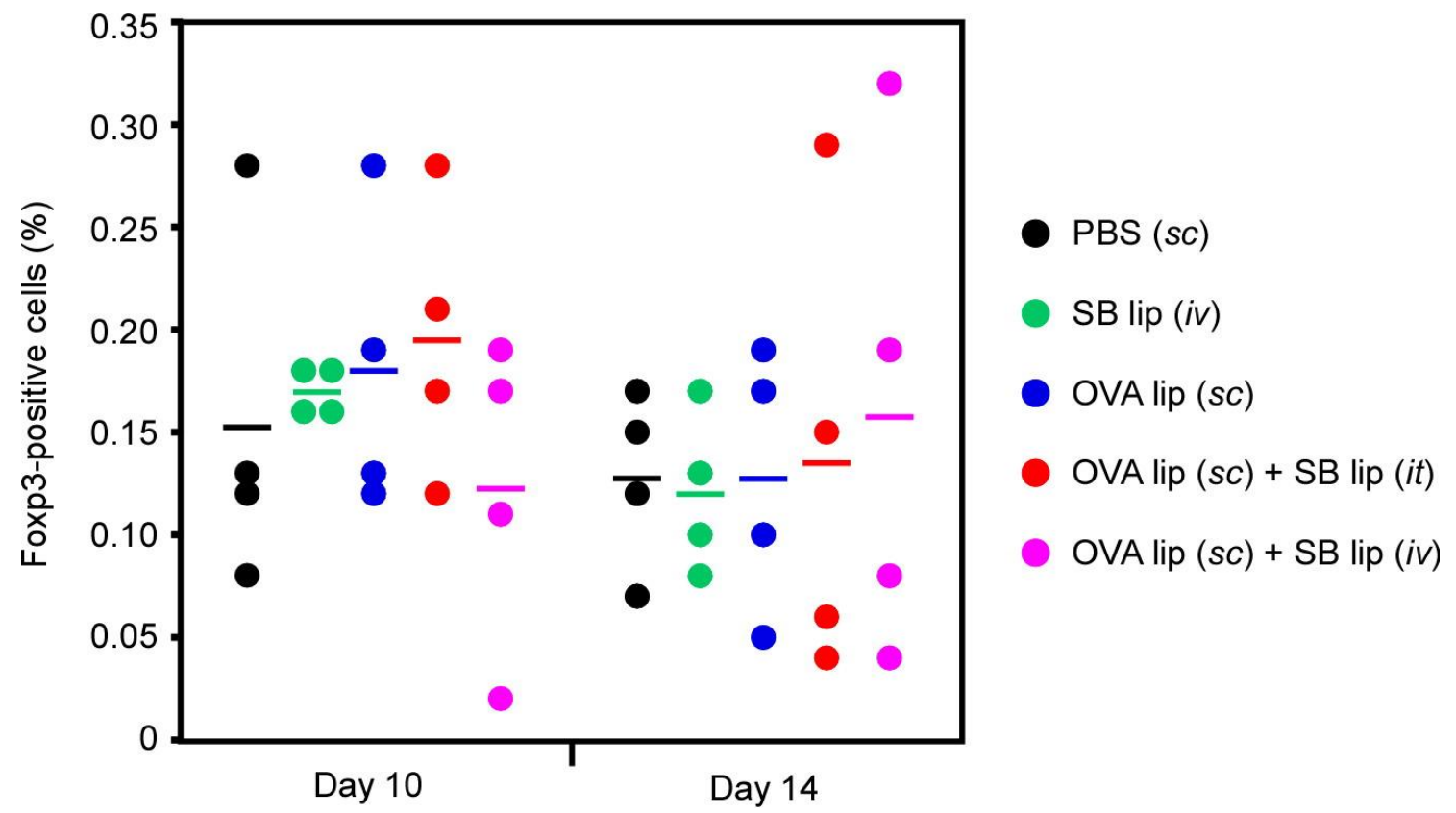

Figure 5. Effect of administration of OVA lip and SB lip on tumor-infiltrating Treg. E.G7-OVA cells $\left(2 \times 10^{6}\right.$ cells/mouse $)$ were transplanted subcutaneously into the left back of each mouse. On day 7, OVA lip containing $10 \mu \mathrm{g}$ of OVA was injected subcutaneously to the back of mice. Then $100 \mu \mathrm{L}$ of SB lip (1.1 mM lipids) was administered intratumorally or intravenously. On days 10 and 14, mice were killed. The tumors were dissected. Then single cells of tumors were prepared. Foxp3-positive cells in tumor-derived cells were detected using flow cytometry after staining with phycoerythrin-labeled Foxp3 antibody.

Figure 6 shows CD8-positive cells in tumor sections, which indicates CD8-positive CTLs in tumors. On Day 10, few CD8-positive cells were observed, irrespective of the treatment group. In contrast, on Day 14, many CD8-positive cells infiltrated into tumor tissue in the cases of OVA lip and/or SB lip-treated mice. Apparently, the combination of intravenous administration of SB lip and OVA lip increased the number of CTLs in tumor tissues. These results were confirmed from flow cytometric analyses of tumor-derived single cells (Figure 7). On Day 14, CD8-positive cells in tumors of the combination of intravenous administration of SB lip and OVA lip were double those in the case of OVA lip. For intravenous administration of SB lip, SB505124 was delivered to CTLs not only in tumors but also in the spleen or in blood circulation considering the biodistribution of PEG-modified liposomes. ${ }^{22,24-26}$ Therefore, intravenous administration of SB lip might suppress the inactivation of CTL more effectively than intratumoral administration does, which engenders the infiltration of more CTLs into the tumor. Although further analysis is necessary to ascertain more details about the induction mechanism of CTL by the combination of OVA lip/SB lip, intravenous administration of SB lip and subcutaneous administration of OVA lip are expected to provide an effective immunity-inducing system for cancer immunotherapy. 
Potentiation of cancer immunity-inducing effect by $\mathrm{pH}$-sensitive polysaccharide-modified liposomes with combination of TGF- $\beta$ type I receptor inhibitor-embedded liposomes

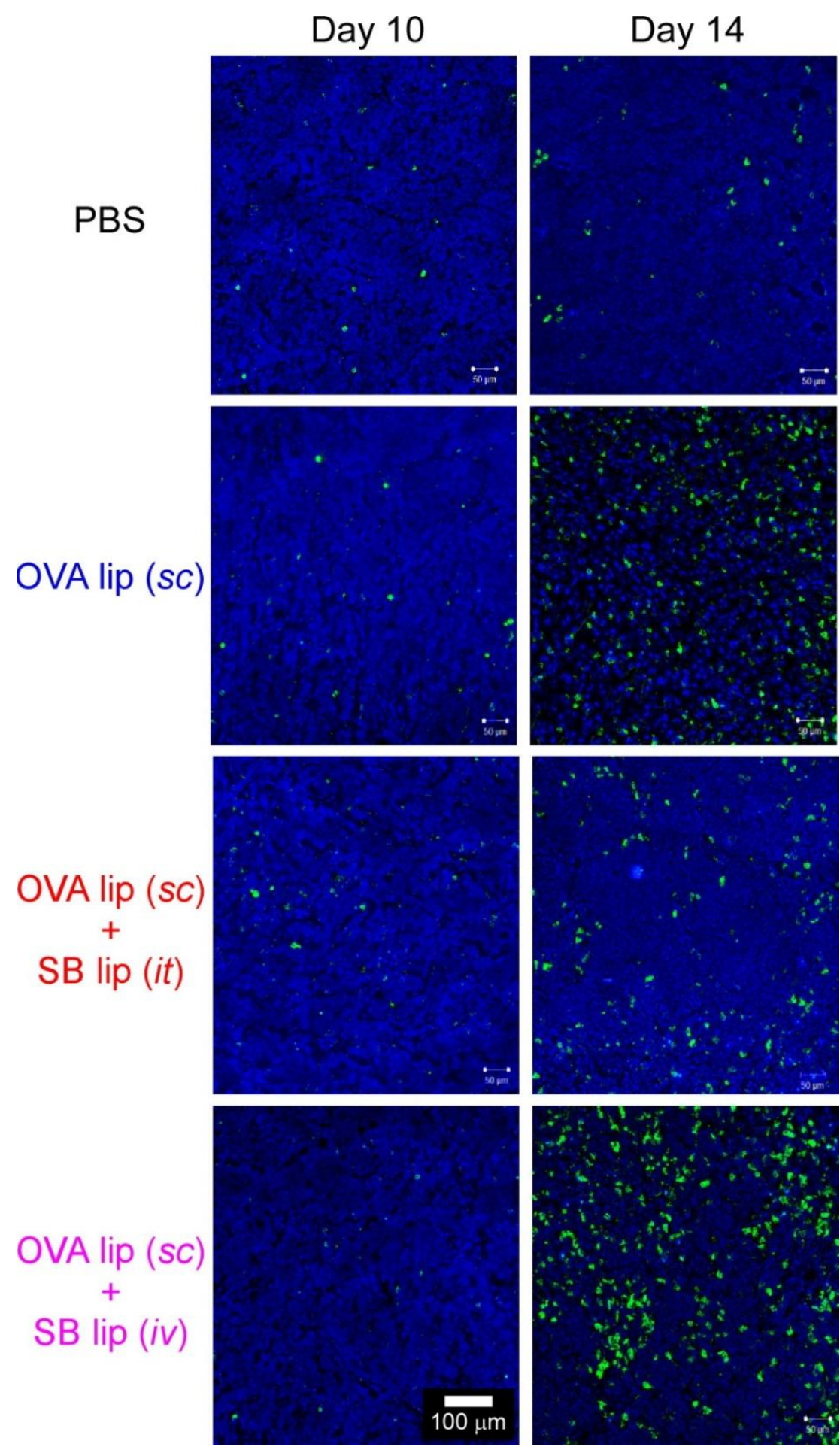

Figure 6. Infiltration of CD8-positive T cells in tumor. E.G7-OVA cells $\left(2 \times 10^{6}\right.$ cells/mouse $)$ were transplanted subcutaneously into the left back of each mouse. On day 7, OVA lip containing 10 $\mu \mathrm{g}$ of OVA was injected subcutaneously into the back of each mouse. Then $100 \mu \mathrm{L}$ of SB lip (1.1 mM lipids) was administered intratumorally or intravenously. On days 10 and 14, mice were killed. Their tumors were dissected. CD8-positive cells in tumor sections were stained with anti-mouse CD8 antibody labeled with Alexa Fluor488 (green) and were observed using CLSM. Cellular nuclei were stained with DAPI (blue). 


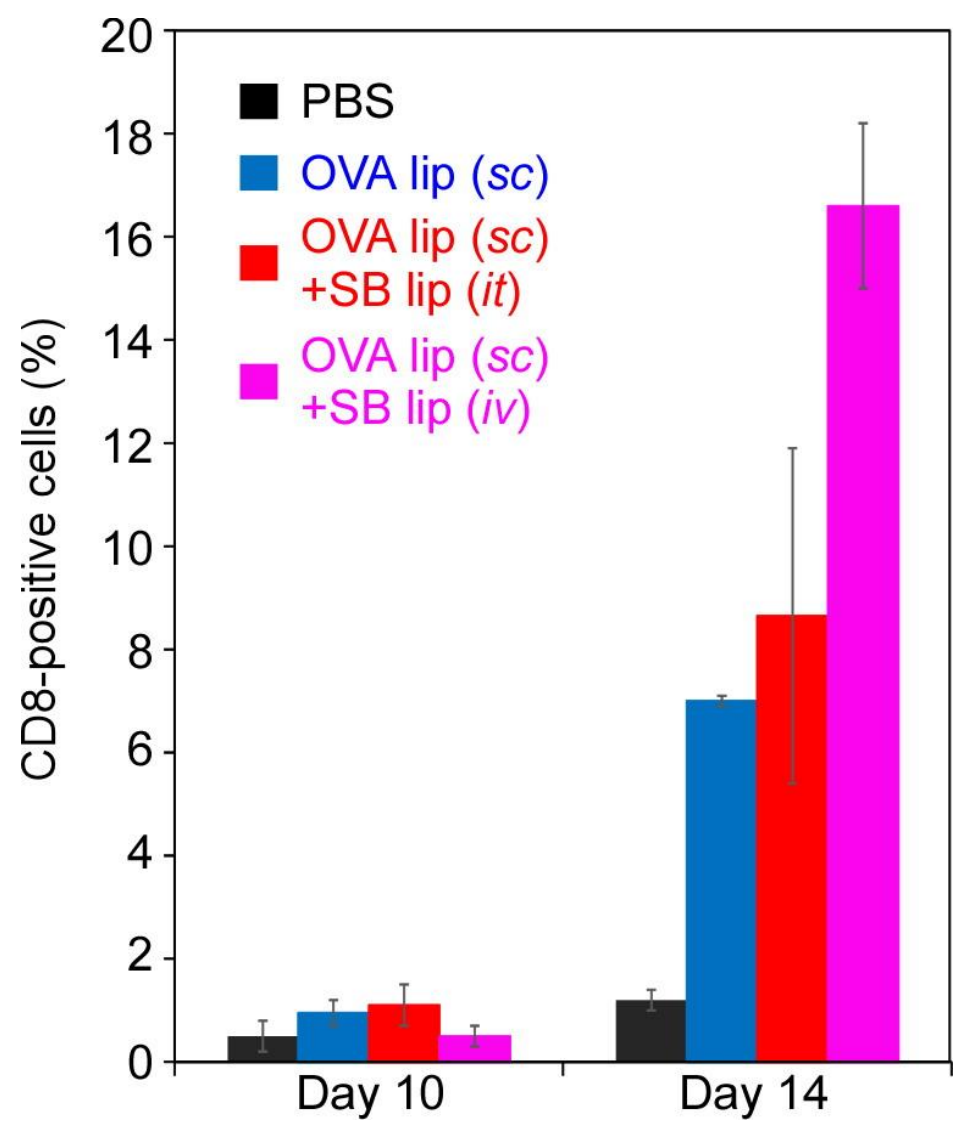

Figure 7. Effects of administration of OVA lip and SB lip on tumor-infiltrating CTL. E.G7-OVA cells $\left(2 \times 10^{6}\right.$ cells/mouse) were transplanted subcutaneously into the left back of mice. On day 7 , OVA lip containing $10 \mu \mathrm{g}$ of OVA was injected subcutaneously into the back of each mouse. Then $100 \mu \mathrm{L}$ of SB lip (1.1 mM lipids) was administered intratumorally or intravenously. On days 10 and 14, after the mice were killed, the tumors were dissected. Single cells of tumors were prepared. CD8-positive cells in tumor-derived cells were detected using flow cytometry after staining with phycoerythrin-labeled CD8 antibody.

\section{Conclusion}

This study investigated the combination of $\mathrm{pH}$-sensitive polysaccharide-modified liposomes for CTL induction and SB505124-embedded liposomes for blocking TGF- $\beta$ signaling. Intravenous administration of SB505124embedded liposomes improved the cancer immunotherapeutic effects induced by $\mathrm{pH}$-sensitive polysaccharide-modified liposomes by promoting the infiltration of CD8-positive $\mathrm{T}$ cells to tumor tissues.
Results show that the combined delivery of antigen and inhibitors of TGF- $\beta$ signaling using proper delivery carriers via appropriate routes is promising for use in potent immunity-inducing systems to achieve both CTL induction and activation.

\section{Acknowledgments}

This work was supported by Grants-in-aid for Scientific Research from the Ministry of Education, Culture, Sports, Science, and Technology of Japan (15H03024, 25750177). 
Potentiation of cancer immunity-inducing effect by $\mathrm{pH}$-sensitive polysaccharide-modified liposomes with combination of TGF- $\beta$ type I receptor inhibitor-embedded liposomes

\section{References}

1. Hodi FS, O'Day SJ, McDermott DF, et al. Improved survival with ipilimumab in patients with metastatic melanoma. $N$ Engl J Med. 2010;363(8):711-723.

2. Topalian SL, Sznol M, McDermott DF, et al. Survival, durable tumor remission, and long-term safety in patients with advanced melanoma receiving nivolumab. $J$ Clin Oncol. 2014;32(10):1020-1030.

3. Tumeh PC, Harview CL, Yearley JH, et al. PD-1 blockade induces responses by inhibiting adaptive immune resistance. Nature. 2014;515(7528):568-571.

4. Flavell RA, Sanjabi S, Wrzesinski SH, and Licona-Limon P. The polarization of immune cells in the tumour environment by TGF $\beta$. Nat Rev Immunol. 2010;10(8):554-567.

5. Gorelik L, Flavell RA. Immune-mediated eradication of tumors through the blockade of transforming growth factor- $\beta$ signaling in T cells. Nat Med. 2001;7(10):1118-1122.

6. Liu VC, Wong LY, Jang T, et al. Tumor evasion of the immune system by converting CD4+CD25- $\mathrm{T}$ cells into CD4+CD25+ T regulatory cells: role of tumor-derived TGF- $\beta . \quad J$ Immunol. 2007;178(5):2883-2892.

7. Connolly EC, Freimuth J, Akhurst RJ. Complexities of TGF- $\beta$ targeted cancer therapy. Int $J$ Biol Sci. 2012;8(7):964-978.
8. Meng $\mathrm{H}$, Zhao $\mathrm{Y}$, Dong $\mathrm{J}$, et al. Two-wave nanotherapy to target the stroma and optimize gemcitabine delivery to a human pancreatic cancer model in mice. ACS Nano. 2013;7(11):10048-10065.

9. Park J, Wrzesinski SH, Stern E, Look M, Criscione J, et al. Combination delivery of TGF- $\beta$ inhibitor and IL-2 by nanoscale liposomal polymeric gels enhances tumour immunotherapy. Nat Mater. 2012;11(10):895-905.

10. Xu Z, Wang Y, Zhang L, Huang L. Nanoparticle delivered transforming growth factor- $\beta$ siRNA enhances vaccination against advanced melanoma by modifying tumor microenvironment. ACS Nano. 2014;8(4):3636-3645.

11. Yuba E. Design of $\mathrm{pH}$-sensitive polymer-modified liposomes for antigen delivery and their application in cancer immunotherapy. Polym J. 2016;48:761-771.

12. Yuba E, Harada A, Sakanishi Y, et al. A liposome-based antigen delivery system using $\mathrm{pH}$-sensitive fusogenic polymers for cancer immunotherapy. Biomaterials. 2013;34(12):3042-3052.

13. Yuba E, Kono Y, Harada A, et al. The application of $\mathrm{pH}$-sensitive polymer-lipids to antigen delivery for cancer immunotherapy. Biomaterials. 2013;34(22):5711-5721. 
Potentiation of cancer immunity-inducing effect by $\mathrm{pH}$-sensitive polysaccharide-modified liposomes with combination of TGF- $\beta$ type I receptor inhibitor-embedded liposomes

14. Yuba E, Tajima N, Yoshizaki Y, et al. Dextran derivative-based $\mathrm{pH}$-sensitive liposomes for cancer immunotherapy. Biomaterials. 2014;35(9):3091-3101.

15. Yuba E, Yamaguchi A, Yoshizaki Y, et al. Bioactive polysaccharide-based $\mathrm{pH}$-sensitive polymers for cytoplasmic delivery of antigen and activation of antigen-specific immunity. Biomaterials. 2017;120:32-45.

16. Banchereau J, Steinman RM. Dendritic cells and the control of immunity. Nature. 1998;392(6673):245-252.

17. Mellman I, Steinman RM. Dendritic cells: specialized and regulated antigen processing machines. Cell. 2001;106(3):255-258.

18. DaCosta-Byfield S, Major C, Laping NJ, Roberts AB. SB-505124 is a selective inhibitor of transforming growth factor- $\beta$ type I receptors ALK4, ALK5, and ALK7. Mol Pharmacol. 2004;65(3):744-752.

19. Moore MW, Carbone FR, Bevan MJ. Introduction of soluble protein into the class I pathway of antigen processing and presentation. Cell. 1988;54(6):777-785.

20. Shen Z, Reznikoff G, Dranoff G, Rock KL. Cloned dendritic cells can present exogenous antigens on both MHC class I and class II molecules. I Immunol. 1997;158(6):2723-2730.
21. Mukherjee S, Ghosh RN, Maxfield FR. Endocytosis. Physiol Rev. 1997;77(3):759-803.

22. Barenholz Y. Doxil ${ }^{\circledR}$ - the first FDA-approved nano-drug: lessons learned. $J$ Control Release. 2012;160(2):117-134.

23. Guo CL, Yang XH, Cheng W, et al. Expression of Fas/FasL in CD8+ $\mathrm{T}$ and CD3+ Foxp3+ Treg cells--relationship with apoptosis of circulating CD8+ T cells in hepatocellular carcinoma patients. Asian Pac J Cancer Prev. 2014;15(6):2613-2618.

24. Maeda H, Wu J, Sawa T, et al. Tumor vascular permeability and the EPR effect in macromolecular therapeutics: a review. $J$ Control Release. 2000;65(1-2):271-284.

25. Wong $\mathrm{AD}$, Ye M, Ulmschneider MB, Searson PC. Quantitative analysis of the enhanced permeation and retention (EPR) effect. PLoS One. 2015;10(5):e0123461.

26. Allen TM, Hansen C, Martin F, et al. Liposomes containing synthetic lipid derivatives of poly(ethylene glycol) show prolonged circulation half-lives in vivo. Biochim Biophys Acta. 1991;1066(1):29-36. 